\title{
The journal develops
}

The fetal and neonatal edition of the journal was introduced five years ago and the number of papers submitted to that section of the journal has increased in number, quality, and variety. Acceptance of any paper for publication depends on the same criteria of originality, scientific reliability, and importance. The publication of the fetal and neonatal edition only quarterly resulted in some papers being included in the standard edition or an unacceptable delay. From this month, the fetal and neonatal edition will be published every alternate month which will enhance the speed of publication and will provide the complete spectrum of papers we are receiving for our increasingly wide readership of obstetricians and geneticists as well as paediatricians.

An increasing number of our readers work as paediatricians either wholly or partly in the community and reviews at our yearly editorial committee meeting have shown that each standard edition of the journal contains about seven papers of special interest to this group of paediatricians. As editors, we aim to ensure that each reader will find at least half a dozen articles of interest in each issue of the journal and our aim to provide a balance between review and original articles helps to achieve this objective. We no longer expect every article to be of interest to every reader and this allows us to accept papers of high scientific quality that appeal to a minority of readers. A series of review articles provides us with an opportunity to publish material in depth and the recent series on types of treatment in child psychiatry will form the beginning of a new venture into book publishing.

All clinicians are now involved in clinical audit and there is a temptation to submit every audit study for publication. These papers are assessed in a similar way to other original articles but we are becoming increasingly stringent in the criteria used for acceptance to avoid the publication of material that is only of local interest or importance. We hope to be in a position shortly when we shall only accept audit papers where the 'loop is closed'. In that type of study there is a description not only of the present imperfections but a reassessment after the introduction of change.

The abstract is the display window of a paper-indeed it may be the only part of the paper that is read particularly if the subject is specialised and appeals to only a minority of readers. Yet many papers we receive have poorly written abstracts. We have resisted pressure to conform to structured style abstracts because they are dull to read. Abstracts must be structured in content and should include, within the allotted number of words, the purpose of the study, what was done, the key results, and a concluding sentence or two. This challenge is well worth taking seriously. Inability to summarise a study succinctly might mean that the study itself lacked direction. Referees often scan the abstract first. Finally you may find the contents of your paper reproduced in journals that rely solely on the abstract. 